Cita bibliográfica. Mejía Vázquez, R., Serrano-Barquín, R.C., López Carré, E. y Serrano-Barquín, H.P. (2017). Participación de las mujeres en empresas de hospedaje del municipio de Toluca, México. Análisis de su empoderamiento. Investigaciones Turisticas (14), pp. 21-44. https://dx.doi.org/10.14461/INTURI2017.14.02

\title{
Participación de las mujeres en empresas de hospedaje del municipio de Toluca, México. Análisis de su empoderamiento
}

\author{
Women's participation in Toluca hosting companies. \\ Analysis of their empowerment
}

Rebeca Mejía Vázquez. Universidad Autónoma del Estado de México. mejiarbk@gmail.com Rocío del Carmen Serrano-Barquín. Universidad Autónoma del Estado de México. rocioserba14@gmail.com Elizabeth López Carré. Universidad Autónoma del Estado de México.delfos311@hotmail.com Héctor Paulino Serrano-Barquín. Universidad Autónoma del Estado de México. hectorsb2012@yahoo.com

\section{RESUMEN}

Los estudios sobre la situación de la mujer mexicana en el sector turístico son escasos, principalmente acerca de su empoderamiento. En este tenor, en el presente artículo se analiza el nivel de empoderamiento de aquellas mujeres que trabajan en el sector hotelero de la ciudad de Toluca, Estado de México. Los datos fueron extraídos de 10 empresas de hospedaje de entre una y cinco estrellas, con una muestra de 102 mujeres que participan laboralmente en ellas. La investigación fue realizada desde la perspectiva de género y con un enfoque cuantitativo. Además, mediante la adición de siete factores se obtuvo la calificación total de empoderamiento y con base en ello se clasificó en alto, medio o bajo, de acuerdo con el rango obtenido. Finalmente, dentro de los resultados se obtuvo que sí se presenta cierto nivel de empoderamiento en la mujer que participa laboralmente en las empresas de hospedaje de Toluca a pesar de factores como el puesto que ocupan y el ingreso que perciben.

Palabras clave: Mujeres, empoderamiento, empresas de hospedaje, Toluca, México.

\section{ABSTRACT}

Studies about the mexican women's situation in the tourism sector, mainly on their empowerment, are limited. For that reason, in this article the empowerment level of women who work in the hospitality industry of Toluca City, State of Mexico, is analyzed. Data were collected 
from ten hosting companies from one to five stars, with a sample of 102 women who work in such places. The research was carried out from gender perspective and with a quantitative approach. Moreover, it was considered the sum of seven factors to obtain the total score of empowerment and then it was ranked as high, medium or low. Finally, as a results, it was observed that there is a certain level of women's empowerment in spite of factors such as, the position in which they are and the income that they earn.

Keywords: Women, empowerment, hosting companies, Toluca, Mexico.

\section{INTRODUCCIÓN}

La mujer es considerada un pilar fundamental en la estructura socioeconómica de la sociedad, participando en el desempeño de las tareas del hogar y el cuidado de la familia (Rico y Gómez, 2009), con los efectos positivos y negativos que ello implica; no obstante, en muchos casos se le ha relegado a su espacio privado sin posibilidad de participar fuera de casa en otras actividades productivas, y es así que a lo largo de los últimos años, se han tenido que redoblar esfuerzos para revertir la exclusión social que vive para dejar de ser concebida como un ser inferior, vulnerable y sin capacidad de ejercer sus derechos (UNESCO, 1955; Daros, 2014).

En este contexto, es a través de diversos acontecimientos en la historia ${ }^{1}$, como se contribuye a dar vida, presencia y protagonismo a un conjunto de mujeres líderes en Europa y América (OIT, 2009). Este activismo a favor de los derechos de las mujeres ha roto barreras y, en las últimas décadas, se ha logrado la incorporación de mujeres en labores tradicionalmente masculinas, dentro del empresariado turístico y en otras actividades donde se suelen continuar ciertos estereotipos y determinismos de sus roles sociales que permiten ubicar a miles de ellas como camaristas, cocineras, afanadoras y otras que dan empleo de baja percepción a quienes permanecen dentro de dichos estereotipos.

Otro aspecto que favorece el mejoramiento de la situación de la mujer en diversos ámbitos independientes o no determinantes de esos roles femeninos históricos, es su paulatina incorporación al mercado laboral (Parada, 2016; Vega, 2016) y su ascenso en la escala corporativa (Contreras et al., 2012). No obstante, siguen existiendo "barreras no explícitas" que obstruyen a las mujeres desempeñar funciones de dirección y mando (Bucheli y Sanroman, 2005); así como la invisibilidad femenina en los estudios en el ámbito ejecutivo laboral (Brunet y Santamaría, 2016).

En el caso de México, la participación laboral femenina en 1990 fue de 36\% (Banco Mundial, 2016), en el 2000 de 29.9\%, 2010 de 33.3\% (INEGI, 2011) y de acuerdo con la Organización para la Cooperación y el Desarrollo Económico (OCDE) en el 2012 fue de 48\%; no obstante, esta última cifra es la tasa más baja con relación al promedio de dicha organización que fue de $62 \%$. En este contexto, su participación en el sector turístico se ha incrementado últimamente; de acuerdo con el Tercer Informe de Gobierno Federal 2014-2015 (Presidencia de

\footnotetext{
${ }^{1}$ Ejemplo de acontecimientos en la historia: durante la Revolución Industrial se presentan movimientos de obreras que luchan por derechos laborales, la lucha contra la esclavitud, el alcoholismo, la explotación de mujeres y niños, y en el caso de México, la guerra de Independencia (1810-1821) con la participación de Josefa Ortiz de Domínguez y Leona Vicario y las soldaderas y revolucionarias como Carmen Serdán en la Revolución Mexicana (1910-1920).
} 
la República, 2015), 57\% de las personas que conforman la fuerza laboral del sector son mujeres; sin embargo, existe escasa información en el Estado de México sobre su situación en este rubro y su empoderamiento. Ante dichas circunstancias, el objetivo de este artículo es analizar el nivel de empoderamiento de la mujer que participa en empresas de hospedaje del municipio de Toluca, Estado de México, desde la perspectiva de género y con un enfoque cuantitativo; ya que existen pocos estudios que incorporen esta perspectiva en el ámbito iberoamericano (Ferguson, 2010; Vizcaíno et al., 2014), igualmente son escasos los resultados positivos donde se han aplicado las políticas de inclusión social para mujeres (UNWTO, 2011; Ferguson, 2011; Ochman, 2016).

El artículo se compone de los siguientes apartados: en el primero se presentan las etapas de desenvolvimiento de la mujer, es decir, los roles que históricamente ha desempeñado, así como su participación en la sociedad para ser valorizada y las conceptualizaciones que la han segregado como resultado de los arraigados estereotipos femeninos y las diferenciaciones en la identidad de género. En este apartado también se presentan los antecedentes acerca de su participación en el ámbito laboral, principalmente en el sector turístico. En el segundo punto se mencionan los fundamentos teóricos que dan pie a la discusión, los cuales giran en torno al empoderamiento de la mujer. Posteriormente, se expone la metodología del trabajo de investigación, seguida de los resultados sobre el nivel de empoderamiento de la mujer que trabaja en hoteles de la capital del Estado de México, y el último apartado está dedicado a las conclusiones.

\section{LA MUJER EN LA SOCIEDAD}

Para tener un panorama general de cómo ha evolucionado la perspectiva de género y la importancia que tiene en el desarrollo de la mujer, se presenta una breve revisión histórica del surgimiento de esta perspectiva de análisis.

En el libro El segundo género de Simone de Beauvoir, publicado en 1949, la autora señala que la mujer no nace, se hace, dando a entender con esta afirmación que la forma de ser y estar de las mujeres en la sociedad está dada en función de lo que aprende de los demás (Lamas, 1986; Cobo, 2014). Este aprendizaje sobre el género y su "actuación", que diversas teóricas del feminismo la denominan como performatividad, activa las diferencias de género desde la primera infancia:

"[...] el niño en desarrollo [...] encuentra generalmente sus principales modelos en los padres [...] ciertos rasgos humanos han sido socialmente especializados para constituir las actitudes y la conducta apropiadas para cada género. Esta especialización social se ha racionalizado entonces en una teoría: la conducta que la sociedad impone es natural para uno de los géneros y anormal para el otro, y el inadaptado lo es a causa de un defecto (glandular o accidental), (Mead, 2013: 276 y 277)."

Al desarrollar la idea del performance de la identidad femenina, varias autoras parten de cuestionamientos de las primeras feministas de los setenta del siglo XX; al respecto, Marta Lamas (2003 y 2014), se había preguntado hasta dónde el género puede ser elegido. Partiendo de la idea de que las personas no sólo somos construidas socialmente, sino que, en cierta medida, las mujeres nos construimos a nosotras mismas, Lamas refiere a su vez a la teórica Judith Butler, 
quien perfila al género como el resultado de un proceso mediante el cual las personas recibimos significados culturales

"el orden simbólico de género está tan profundamente arraigado que no requiere justificación: se impone a sí mismo como autoevidente, y es considerado como 'natural' gracias al acuerdo casi inmediato y perfecto que obtiene de estructuras sociales tales como la organización social del espacio y tiempo y la división sexual del trabajo (Lamas, 2014:160)."

Estos procesos de construcción sociocultural de lo que es lo femenino y lo masculino, genéricamente hablando, son condicionamientos que acompañan a los individuos desde su nacimiento hasta en las aparentes insignificancias de la crianza infantil, que se reflejan en las diversas posibilidades de comportamiento, tanto explícita como tácitamente determinadas para los hijos e hijas, pues ya están establecidas "las maneras de identificarlos a partir de la vestimenta, los arreglos y poses corporales, según los patrones sociales de masculinidad y feminidad" (Núñez, 1999: 54). Es en esas etapas en que los mismos se vinculan por separado con la madre ("ama de casa") o el padre ("proveedor" histórico), ocasionando que todo ello derive en procesos complejos empleados para la representación de los roles sociales, incluidas las conceptualizaciones sobre lo que la mujer debe desempeñar laboralmente. La importancia que en este contexto tiene la construcción de la identidad sexual implica que, a partir de ella, se cristalizan discursos, símbolos, concepciones e ideas sobre determinados comportamientos y roles sociales identitarios que se inician con la diferenciación entre los géneros e impacta la vida futura de las personas, incluyendo los trabajos o tareas que se consideran "correctos" para cada mujer o varón de acuerdo con su rol social de género. Es así que "la acción (esto es, la interpretación del rol) se relaciona con una estructura definida por la diferencia biológica, la dicotomía masculino/femenino y no con una estructura definida por las relaciones sociales... la polarización es una parte necesaria del concepto" (Connell, 2015: 56), esta polarización o radicalización es ancestral y ha provocado, entre otros factores sociológicos, lo que se conceptualizó como la "División sexual del trabajo", que históricamente determinó discriminación y exclusión de las mujeres que buscaron en el empleo una forma de mejoría en sus condiciones de desarrollo, por lo que se indagaron las nuevas formas de empoderamiento femenino que surgen en las décadas recientes.

La lucha por los derechos de la mujer se ha llevado a cabo de diferente manera, continuando hasta la actualidad (Lagarde, 2003). En este tenor, en la Tabla 1 se muestran las etapas iniciales de su participación en la sociedad. Los autores identifican tres etapas correspondientes a este proceso, resaltando aspectos que le han permitido romper con las "limitaciones" y paradigmas sobre lo que debe hacer. Además, se mencionan hechos acerca de cómo se ha desenvuelto en la sociedad, los roles que ha desempeñado y su situación respecto a la del hombre. Por ejemplo, de acuerdo con Lipovetzky (en Daros, 2014), en la primera etapa, al hombre se le atribuyeron valores superiores en relación con la mujer, situación que cambió en los años siguientes. Por otro lado, Cobo (2014) menciona cómo la mujer fue teniendo mayor participación en la sociedad, por ejemplo al obtener derechos como ciudadanas. En la actualidad la participación de la mujer en la sociedad continúa evolucionando y hay quien ya incorpora una "cuarta ola" feminista (Matos y Paradis, 2013). 
Cabe destacar que el proceso de incorporación de la mujer en la sociedad ha sido paulatino y a través de los años ha incrementado su participación en diversos ámbitos, tal es el caso del laboral, político, social, entre otros; sin embargo, en algunos no ha sido totalmente aceptada.

Tabla 1. Principales etapas de desenvolvimiento de la mujer

\begin{tabular}{|c|c|c|c|}
\hline Autor & Lipovetzky (en Daros, 2014) & $\begin{array}{l}\text { Periodo- } \\
\text { etapa }\end{array}$ & Cobo (2014) \\
\hline $\begin{array}{l}\text { Antes del siglo XII- } \\
\text { Primera Mujer }\end{array}$ & $\begin{array}{l}\text { Distribución de roles de forma } \\
\text { asimétrica; al hombre se le } \\
\text { atribuyeron valores superiores en } \\
\text { contraste con la mujer que se } \\
\text { estimaron de inferiores. Existía } \\
\text { desvalorización y desprecio hacia } \\
\text { ellas }\end{array}$ & $\begin{array}{l}\text { Siglo XVII- } \\
\text { Primera ola feminista }\end{array}$ & $\begin{array}{l}\text { La subordinación radica en la } \\
\text { sociedad y no en la naturaleza. } \\
\text { Reclamación de sus derechos de } \\
\text { ciudadanía (en la Revolución } \\
\text { Francesa). } \\
\text { Se mantienen las estructuras } \\
\text { patriarcales }\end{array}$ \\
\hline $\begin{array}{l}\text { Siglos XII-XVIII- } \\
\text { Segunda mujer }\end{array}$ & $\begin{array}{l}\text { En esta etapa, la mujer fue exaltada; } \\
\text { tal es el caso en las épocas del } \\
\text { Renacimiento e llustración. No } \\
\text { obstante, la situación de la mujer } \\
\text { siguió confinada al hogar aunque } \\
\text { existía cierta influencia de ella sobre } \\
\text { el marido }\end{array}$ & $\begin{array}{c}\text { Siglo XIX- } \\
\text { Segunda ola feminista }\end{array}$ & $\begin{array}{l}\text { Participación de la mujer en el } \\
\text { movimiento sufragista. Este } \\
\text { movimiento finaliza con la I } \\
\text { Guerra Mundial. Además, en } \\
\text { ese siglo, las mujeres se } \\
\text { articulan en torno al derecho al } \\
\text { voto }\end{array}$ \\
\hline $\begin{array}{c}\text { Mediados del siglo } \\
\text { XX- } \\
\text { Mujer } \\
\text { indeterminada o } \\
\text { posmujer }\end{array}$ & $\begin{array}{l}\text { Pierde fuerza la idea de que la mujer } \\
\text { solo debe estar en casa. Otra } \\
\text { característica de esta etapa es que la } \\
\text { mujer puede elegir lo que desee ser }\end{array}$ & $\begin{array}{c}\text { Siglo XX- } \\
\text { Tercera ola feminista }\end{array}$ & $\begin{array}{l}\text { Simone de Beauvoir explicó que } \\
\text { la mujer es naturaleza (crean } \\
\text { vida biológica) y el hombre es } \\
\text { cultura (crean vida social y } \\
\text { política). } \\
\text { Las mujeres fueron empujadas a } \\
\text { aceptar su identidad }\end{array}$ \\
\hline
\end{tabular}

Fuente: Elaboración propia con base en Lipovetzky (en Daros, 2014) y Cobo (2014).

Es a partir de 1950 cuando se incrementa visiblemente la presencia de la mujer en el ámbito laboral en los países de Europa. Por otra parte, naciones como Suecia y México consideran el doble papel (madre y trabajadora) que juega la mujer en la sociedad (Loggia, 1998).

A lo largo del tiempo, la participación de la mujer en el ámbito laboral ha presentado altibajos que influyen en la existente desigualdad de género, a pesar que se ha ido posicionando de manera paulatina en diferentes sectores, no ha sido suficiente para lograr un empoderamiento femenino. Y resulta que las "barreras no explícitas" (techo de cristal) son una superficie superior invisible en el aspecto laboral de las mujeres, estas barreras impiden que ellas sigan avanzando a puestos de mayor jerarquía (Bustos, 2007; Roldán et al., 2012) y cuanto más altos son los cargos de responsabilidad en la sociedad, menos mujeres vemos; por ejemplo, el porcentaje de mujeres que gobiernan un país es menor a 10\% (Botello, 2014). En este contexto, los propios partidos políticos argumentan que las mujeres no están interesadas en la política, situación que se utiliza para explicar por qué no están igualmente representadas en las instituciones, puestos de poder y en los mismos partidos que los hombres (UNWomen, 2014: 31). De manera paralela, y con base en datos de la UNWomen (2015), en el ámbito laboral, 50\% de las mujeres trabaja y recibe un 
salario; sin embargo, perciben menos dinero (entre 10 y $30 \%$ ) que los hombres, aunque desarrollen las mismas actividades.

Cabe mencionar que, hoy en día, las mujeres están ingresando en ámbitos que eran ocupados tradicionalmente por hombres (Moreno y Lunar, 2006; Baum, 2013; Aguilar et al., 2015), tal es el caso del sector turístico. En este sentido, el empleo en este sector está organizado en función del género, por tal motivo, los salarios de las trabajadoras son bajos (Ferguson y Moreno, 2014). Es así que, el turismo, como estrategia de desarrollo, ha recibido poca atención al respecto, en especial sobre la justicia social global y el combate contra las desigualdades (Ferguson, 2010).

Según Hernández et al. (2014: 116), en el Estado de México, es posible observar un escenario que presenta dinámicas de poder tradicional, donde las mujeres se han incorporado paulatinamente como actores políticos; no obstante y a pesar de su presencia en cargos públicos relacionados con turismo, no se corresponde con su participación en el mercado laboral en ese sector; pese a su creciente incorporación cuantitativa al sector público, la capacidad femenina se ve frenada de actuar y ejercer su poder, acercándose más al rol masculino.

De acuerdo con la OMT (2010), el sector turístico posee un gran efecto multiplicador, es más propenso a contratar mujeres que en otros sectores; ya que, en empleos como ayudantes de concina o limpieza, no se requiere de una preparación profesional, pues reflejan las actividades que cotidianamente realizan, además, permite desarrollar negocios turísticos en los propios hogares y el horario puede ser flexible, lo que contribuye para que las mujeres atiendan también responsabilidades domésticas. Aunque este organismo señala que existen datos poco exactos sobre la participación de las mujeres en el ámbito laboral turístico internacional (de los 172 países estudiados en el informe anterior, solo 101 contaban con datos), las mujeres representan un mayor porcentaje al respecto, se estima que en los países en desarrollo $60 \%$ de ellas se desempeñan en este ámbito, principalmente en áreas administrativas. Por otro lado, se han publicado artículos que proporcionan información en torno a la situación laboral de las mujeres en proyectos turísticos (Baum, 2013; Legazpe, 2015; Brunet y Santamaría, 2016; Parada, 2016; ONU, 2016; Vega et al., 2016); pero como señala Ferguson (2010), son pocos los trabajos enfocados a incorporar la perspectiva de género que visibilice las desigualdades de género, etnia y otras variables.

A nivel nacional, el turismo cuenta con una de las mayores participaciones dentro de la economía de la nación, con $8.7 \%$ del PIB en el 2013, junto con las remesas, la producción industrial y la producción de petróleo. Además, es el segundo sector con mayor participación de mujeres, debido a que representan $57 \%$ del total de personas ocupadas (Presidencia de la República, 2015). En el Estado de México, la producción bruta de las actividades turísticas en el 2013 fue del 7.4\%, siendo el tercer estado mexicano con mayor aportación (INEGI, 2015).

De acuerdo con la Encuesta Nacional de Ocupación y Empleo (ENOE) [Gobierno del Estado de México, 2011], la participación de la mujer mexiquense2 en el mercado laboral fue de 38\%, lo que ubica al Estado en la vigesimosexta posición a nivel nacional al tener bajos índices de empleo

\footnotetext{
${ }^{2}$ Gentilicio del Estado de México
} 
femenino. Por otra parte, acerca de la situación sobre empresas de hospedaje en el Estado de México, se tiene registro de 1,040 Unidades Económicas de servicios de alojamiento temporal, con un total de personal ocupado de 10,947 , del cual $55.31 \%$ son mujeres. En relación con los puestos que ocupa, el mayor número se inclina hacia áreas de producción, ventas y servicios, mientras que los hombres ocupan en mayor medida cargos administrativos, contables y de dirección total. Cabe destacar que del personal que trabaja por honorarios o comisiones sin sueldo base, $38.6 \%$ son mujeres (INEGI, 2014b).

En el caso del municipio de Toluca, capital del Estado de México (ver Figura 1, localización geográfica), la población total de mujeres económicamente activas es de 122,702. Por otra parte, de la población ocupada, 22,696 laboran en la actividad productiva de servicios, lo que representa $18.5 \%$ de las mujeres empleadas (H. Ayuntamiento de Toluca, 2013). Al contrario de los datos estatales, la mujer y el hombre que vive en el municipio de Toluca tienen la misma participación en áreas administrativas o de dirección y es en áreas de producción, ventas o servicios donde la mujer tiene mayor participación con un $58 \%$. No obstante, con estas cifras y datos presentados no se conoce con detalle la situación de la mujer en el sector turístico en la ciudad de Toluca ni sobre su empoderamiento. Por tal motivo, esta investigación es un primer acercamiento a estos temas, con el fin de ampliar y dar continuidad a los estudios relacionados con el nivel de empoderamiento, que como ya se expuso, existen pocos trabajos en el ámbito latinoamericano que permitan establecer políticas enfocadas a mejorar las condiciones laborales de las mujeres y seguir avanzando hacia una sociedad más equitativa.

Figura 1. Localización geográfica de Toluca, México

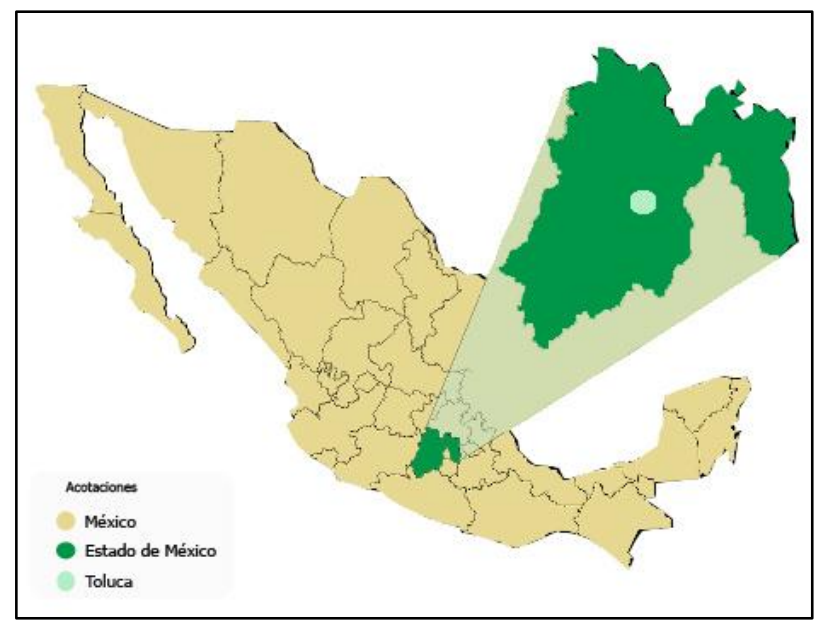

Fuente: Elaboración propia con base en INEGI (2014a)

\section{GÉNERO Y EMPODERAMIENTO}

Los conceptos que dan sustento teórico al artículo son los de género y empoderamiento. En relación con el primero, se entiende como una estructura de poder y "una categoría que da cuenta de una forma de organizar jerárquicamente las relaciones entre hombres y mujeres en cada sociedad" (Cobo, 2014: 10). 
En este sentido, la OMT (2013) y Moreno y Lunar (2006) señalan que la mayoría de los conceptos coincide en que el género es un rol asignado por las sociedades, igualmente éste determina las responsabilidades, personalidad y el sistema de valores y creencias de una persona en función del sexo. De igual forma, implica una serie de conductas preestablecidas tanto en unos como en otras (Araiza, 2004: 136). Otros autores, como Contreras, et al. (2012) añaden, además del factor social, el contexto histórico.

Rosa Cobo (2014), menciona que el significado de género hace referencia a los preceptos que tienen las mujeres dentro de las sociedades patriarcales. De igual forma, las características del género femenino se relacionan con la maternidad, el trabajo doméstico, los cuidados y la ausencia de poder. Por lo que cada sociedad "produce un modo específico de ser mujer"; es así que de alguna manera, al ser obligatorio, la mujer tiende a aceptarlo. En este contexto, el Patriarcado, es un sistema de dominio masculino que mantiene a las mujeres subordinadas de acuerdo con Kate Millet (1995 en Cobo, 2014), promotora del movimiento feminista en la década de los 60's.

En 1990 Walby (en Fernández y Martínez, 2010) argumentó que una de las estructuras de control sobre las mujeres en las sociedades patriarcales se encuentra en la esfera laboral, reflejándose en el impedimento y limitaciones de las mujeres para realizar ciertas tareas así como su baja percepción salarial. Por otro lado, Fernández y Martínez (2010), Cacique (2008), Gutiérrez y Limas (2008), Pacheco (2012) y Caamaño, (2010) afirman que la segregación laboral se fundamenta en el supuesto de que existen "trabajos de hombres" y "trabajos de mujeres", siendo el género la razón por la que estos últimos son menos valorados.

A finales del siglo XX, se comenzó a usar el término "techos de cristal" para denominar a las barreras "invisibles", que dificultan el acceso de las mujeres a los puestos de mayor poder, prestigio o salario, en cualquier ámbito laboral y país (Guil, 2008: 213). En México, las mujeres enfrentan obstáculos que les impiden participar plenamente en el mercado laboral como: la carga del trabajo no remunerado, los tradicionales roles de género y la carencia de políticas de conciliación entre trabajo y vida familiar (OCDE, 2012). Por otra parte, las mujeres no sólo se enfrentan a esta situación sino también a lo conocido como "suelo pegajoso", refiriéndose a las fuerzas que mantienen a las mujeres en trabajos menos importantes (Martín-Llaguno y NavarroBeltrá, 2015), con condiciones laborales precarias, muchas veces considerados como puestos para mujeres (Díaz, Verján y Castrejón, 2014), de baja responsabilidad y, por tanto, de menor salario (Wirth, 2001).

En este contexto, "el concepto de empoderamiento no es nuevo" (Charlier y Caubergs, 2007:9); por ejemplo, los movimientos feministas reivindican desde 1985 la noción de empoderamiento como toma de "poder", resaltando el fortalecimiento de la autoestima y la confianza en sí mismo (Camacho, Martínez y Robert, 2003); además, se vincula con el poder colectivo de cambio de las relaciones de género en los diferentes sectores: económico, político, sociocultural, entre otros (Charlier y Caubergs, 2007); también se considera un proceso complejo (Sosme y Casados, 2016) y que se refleja en el entorno familiar y social de la mujer, por lo que tendrá un efecto positivo en el desarrollo de su comunidad (Murguialday, 2006). 
El empoderamiento es "un proceso multidimensional de carácter social" (Page y Czuba, 1999 en Fang, 2011: 29), mediante el cual las personas ganan el control sobre sus propias vidas, la ideología y los recursos que determinan el poder (Araiza, 2004). Por otra parte, Rappaport (1981 en Cano y Arroyave, 2014) añade que el control de la vida propia se gana "con un entendimiento crítico de su entorno" y propone dos niveles de empoderamiento: individual y colectivo; basados en prácticas dirigidas al desarrollo, la auto-organización y el bienestar social (Colletis y Pecqueur, 1995; Mendizabal y López, 2013). En este contexto, en la investigación se analizó un empoderamiento individual.

Aunado a lo anterior, los procesos de empoderamiento se dan a través de tres aspectos: la educación, el trabajo y el liderazgo, características que permiten entrever la contribución de la mujer en la sociedad (Botello, 2014). La educación es probablemente la condición previa más importante para mejorar la posición de las mujeres; sin embargo, no garantiza totalmente su empoderamiento (Hernández y García, 2015). Cabe destacar que el verdadero empoderamiento es el que cada persona logra y encuentra por sí mismo (Delgado et al., 2010).

Aranda (2006 en Fernández y Martínez, 2010: 10), señala que el empoderamiento con base en la perspectiva de género, es "el fortalecimiento de la identidad femenina, el desarrollo de sus capacidades, su desenvolvimiento profesional y personal, sobre todo, el reconocimiento social y el auto-reconocimiento y autonomía". Aunado a lo anterior, el empoderamiento se refiere al aumento en la capacidad de las mujeres para definir sus opciones de vida y tomar sus propias decisiones (Kabeer, 1999). Para que adquieran autonomía, es necesario que se constituyan como individuos, separándose de su rol materno y doméstico. Mientras no logren esto, seguirán siendo dependientes (Pérez y Vázquez, 2009).

En cuanto su empoderamiento en el sector turístico, Chant (en Ferguson, 2010: 127) afirma que "a pesar de las desigualdades en la participación de las mujeres en el proceso de producción turística", los trabajos a los que han tenido acceso, de alguna forma han contribuido en su empoderamiento, debido a que fomenta que las mujeres "actúen por sí mismas, para sí mismas y demanden un trato más justo en sus lugares de trabajo, en el hogar y en el conjunto de la sociedad".

\section{ASPECTOS METODOLÓGICOS}

La investigación que da soporte a este artículo tuvo lugar en las empresas de hospedaje de Toluca, Estado de México, para conocer el nivel de empoderamiento de las mujeres que participan laboralmente en ellas. Los datos sobre los hoteles asentados en la capital del Estado se encontraron en el Directorio de empresas de hospedaje de la Secretaría de Turismo del Estado de México (SECTUR, 2016).

En la investigación de campo se utilizaron dos cuestionarios, el primero con base en Buquet et al. (2013), se aplicó al gerente general o encargado de Recursos Humanos de cada hotel, para conocer la distribución del personal por género en las empresas de hospedaje de Toluca, así como su ingreso mensual; con los datos se obtuvo el universo (138 mujeres) para después calcular la muestra de la investigación. En la Tabla 2 se presenta la distribución del 
personal de los hoteles (por categoría). Recuérdese que el primer acercamiento al análisis de la situación de la mujer parte de la distribución por sexo ${ }^{3}$, posteriormente se visibiliza su situación respecto a los estereotipos que la circunscriben a ciertas actividades, como se verá posteriormente.

Tabla 2. Distribución del personal que trabaja en empresas de hospedaje del municipio de Toluca, México (2016)

\begin{tabular}{|ccccc|}
\hline $\begin{array}{c}\text { Hoteles por } \\
\text { categoría }\end{array}$ & $\begin{array}{c}\text { No. de } \\
\text { hoteles }\end{array}$ & $\begin{array}{c}\text { No. de } \\
\text { empleados } \\
\text { varones }\end{array}$ & $\begin{array}{c}\text { No. de } \\
\text { empleadas } \\
\text { mujeres }\end{array}$ & $\begin{array}{c}\text { Total de } \\
\text { empleados }\end{array}$ \\
\hline $\mathbf{5}^{*}$ & 1 & 13 & 18 & 31 \\
\hline $\mathbf{4}^{*}$ & 5 & 63 & 93 & 156 \\
\hline $\mathbf{3}^{*}$ & 2 & 22 & 22 & 44 \\
\hline $\mathbf{1}^{*}$ & 2 & 4 & 5 & 9 \\
\hline Total & 10 & 102 & 138 & 240 \\
\hline
\end{tabular}

Fuente: Elaboración propia con base en SECTUR Estado de México (2016) e investigación de campo.

El tamaño de la muestra se determinó mediante la fórmula (Fisher y Espejo, 2011):

$$
n=\frac{Z_{\alpha}{ }^{2} * \mathrm{~N} * \mathrm{p} * \mathrm{q}}{i^{2}(N-1)+Z_{\alpha}{ }^{2} * p * q}
$$

Donde:

$\mathrm{N}$ =tamaño de la población o universo (138 mujeres)

$\mathrm{Z}=$ es el nivel de confianza (95\%)

$\mathrm{i}=$ es el error muestral deseado (5\%)

$\mathrm{p}=$ proporción de individuos que poseen en la población las características de estudio (0.5)

$q=$ es la proporción de individuos que no poseen esa característica (0.5)

La muestra fue de 102 mujeres, a quienes se les aplicó el segundo cuestionario, diseñado con base en el instrumento para medir el empoderamiento de la mujer (Hernández y García, 2008), a fin de evaluar el nivel de empoderamiento en mujeres mexicanas; ya que este instrumento fue validado y se aplicó en el contexto mexicano. Cabe destacar que se realizaron modificaciones para efecto de esta investigación, como el orden de preguntas y número de reactivos para adecuarlas a las condiciones específicas del universo estudiado y facilitar la construcción de la base de datos al dejar todos los ítems de manera positiva.

Posterior a la aplicación de los cuestionarios, se construyó la base de datos y se analizó a través del paquete estadístico SPSS; obteniendo en el análisis de fiabilidad un alfa de Cronbach de 0.863 para 27 reactivos, esto indica que la escala es confiable. En el análisis factorial con

\footnotetext{
${ }^{3}$ Tradicionalmente se habla de sexo a la distinción biológica entre hombres y mujeres, en tanto que género se utiliza para englobar la diversidad sexual que se presenta en una sociedad, para este artículo se habla de género para referirse a hombres y mujeres.
} 
rotación Varimax, se extrajeron 7 factores que representan $65.027 \%$ de la varianza total; los cuales se desglosan en la Tabla 3.

Tabla 3. Factores

\begin{tabular}{|c|c|}
\hline FACTOR & CONCEPTO \\
\hline Igualdad & $\begin{array}{l}\text { Representa una mujer con perspectiva de equidad de género, lo que permite } \\
\text { competir y luchar por mejores posiciones. }\end{array}$ \\
\hline Independencia & $\begin{array}{l}\text { Una persona independiente tiene la capacidad de tomar sus propias decisiones, } \\
\text { decidir por sí misma sin depender de otras personas. }\end{array}$ \\
\hline $\begin{array}{l}\text { Influencias } \\
\text { externas }\end{array}$ & $\begin{array}{l}\text { El liderazgo depende en gran medida de la persona misma y no a hechos ajenos a } \\
\text { ella, probablemente con un locus de control interno. }\end{array}$ \\
\hline Liderazgo & $\begin{array}{l}\text { Proceso que ayuda a otros para trabajar con entusiasmo, hacia determinados } \\
\text { objetivos. Un líder debe tener cualidades personales (activa, emprendedora, } \\
\text { autosuficiente y con alta participación en actividades) para influir en las personas. }\end{array}$ \\
\hline $\begin{array}{l}\text { Satisfacción } \\
\text { Social }\end{array}$ & $\begin{array}{l}\text { Se refiere a cómo se percibe la persona con respecto a su entorno social } \\
\text { representa a una persona con alto grado de confianza. }\end{array}$ \\
\hline Seguridad & $\begin{array}{l}\text { Representa a una persona segura de sí misma, con confianza para realizar } \\
\text { acciones y buscar mejores posiciones en cualquier ámbito. }\end{array}$ \\
\hline Temeridad & Persona valiente, sin temor a tomar decisiones, segura en sus acciones. \\
\hline
\end{tabular}

De acuerdo con los resultados obtenidos en el análisis factorial, en la Tabla 4, se aprecian los siguientes rangos (bajo, medio y alto) para cada factor. 
Tabla 4. Rango factores

\begin{tabular}{|c|c|c|c|c|c|}
\hline Factores & \multicolumn{2}{|c|}{ Rango } & Factores & \multicolumn{2}{|c|}{ Rango } \\
\hline \multirow{3}{*}{ Igualdad } & Bajo & $\geq 4 y \leq 8$ & \multirow{3}{*}{$\begin{array}{l}\text { Satisfacción } \\
\text { Social }\end{array}$} & Bajo & $\geq 2 y \leq 4$ \\
\hline & Medio & $\geq 9 y \leq 12$ & & Medio & $\geq 5 y \leq 6$ \\
\hline & Alto & $\geq 13 y \leq 16$ & & Alto & $\geq 7 y \leq 8$ \\
\hline \multirow{3}{*}{ Independencia } & Bajo & $\geq 4 y \leq 8$ & \multirow{3}{*}{ Seguridad } & Bajo & $\geq 6 y \leq 12$ \\
\hline & Medio & $\geq 9 y \leq 12$ & & Medio & $\geq 13 y \leq 18$ \\
\hline & Alto & $\geq 13 y \leq 16$ & & Alto & $\geq 19 y \leq 24$ \\
\hline \multirow{3}{*}{ Influencias externas } & Bajo & $=1$ & \multirow{3}{*}{ Temeridad } & Bajo & $\geq 2 y \leq 4$ \\
\hline & Medio & $\geq 2 y \leq 3$ & & Medio & $\geq 5 y \leq 6$ \\
\hline & Alto & $=4$ & & Alto & $\geq 7 y \leq 8$ \\
\hline \multirow{3}{*}{ Liderazgo } & Bajo & $\geq 8 y \leq 16$ & & & \\
\hline & Medio & $\geq 17 y \leq 24$ & & & \\
\hline & Alto & $\geq 25 y \leq 32$ & & & \\
\hline
\end{tabular}

Posteriormente, se hizo el cálculo de los factores, clasificándolos de acuerdo con su rango en: bajo, medio o alto, considerando que los valores de cada reactivo que integra dicho factor son del 1 al 4 (escala de Likert). En la Tabla 5, se desglosa dicha información. Finalmente, el indicador "empoderamiento" se integró sumando dichos rubros.

Tabla 5. Calificación y categoría de cada factor

\begin{tabular}{ccc}
\hline Factores & Calificación & Categoría \\
\hline Igualdad & 11.4 & Medio \\
\hline Independencia & 10.9 & Medio \\
\hline Influencias externas & 3.1 & Alto \\
\hline Liderazgo & 24.4 & Alto \\
Satisfacción Social & 5.4 & Medio \\
\hline Seguridad & 19.6 & Alto \\
Temeridad & 5.7 & Medio \\
\hline
\end{tabular}

Elaboración propia.

Por lo tanto, el empoderamiento es igual a la sumatoria de igualdad, independencia, influencias externas, liderazgo, satisfacción social, seguridad, temeridad. Teniendo como resultado: $e=80.3$

A partir del número de reactivos y las opciones de respuesta (escala Likert), se asignaron los rangos de empoderamiento para obtener los niveles bajo, medio y alto; como se muestra en la Tabla 6. 
Tabla 6. Clasificación "empoderamiento"

\begin{tabular}{|lcc|}
\hline \multirow{2}{*}{ Factor } & \multicolumn{2}{c|}{ Rango } \\
Empoderamiento & Bajo & $27-53$ \\
\cline { 2 - 3 } & Medio & $54-81$ \\
\cline { 2 - 3 } & Alto & $82-108$ \\
\hline
\end{tabular}

Elaboración propia.

\section{RESULTADOS Y DISCUSIÓN}

Con base en los resultados del primer cuestionario aplicado, se obtuvo que del total de empleados (240 personas) que laboran en los hoteles en Toluca, $57.5 \%$ son mujeres y $42.5 \%$ son hombres; en este sentido, se confirma que la participación de la mujer en el sector hotelero de Toluca es mayor que la del hombre. Situación que se presenta también a nivel nacional con $58.5 \%$ (González, 2013) y a nivel estatal con 55\% (INEGI, 2014).

Por otra parte, de acuerdo con los resultados de la investigación, en la Tabla 7 se observa que, en términos generales, los puestos que mayoritariamente ocupan las mujeres son aquellos cuyas características son acordes a las que históricamente se han asignado al género femenino y que se relacionan con la maternidad, el trabajo doméstico, los cuidados y la ausencia de poder (Cobo, 2014; Méndez, 2008); ya que las mujeres en el sector turismo ocupan puestos considerados "femeninos": camaristas, auxiliar de cocina, recepcionistas, stewards y amas de llaves (Fernández y Martínez, 2010). Estos puestos representan 70\% del género femenino que labora en las empresas de hospedaje de Toluca. Por otro lado, las mujeres ocupan $38 \%$ en el puesto de camarista y sólo 3\% de hombres ocupa el mismo puesto. El segundo puesto con mayor participación de la mujer es el de recepcionista con 13\%, seguido de auxiliar de cocina con $8 \%$ y ama de llaves. Mientras que los hombres se desempeñan principalmente en el área de mantenimiento $(23 \%)$, recepción $(18 \%)$ y como vigilantes $(11 \%)$.

Destacan los puestos de gerente general y recepcionista, en donde se observa una mayor equidad en cuanto al número de personas que desempeñan dichos cargos y esto se asocia a factores como el nivel de escolaridad; en otras palabras, la mujer está mejor preparada para llevar a cabo las diferentes actividades que se requieren en esos puestos, comparando con el estudios de Salgado y Miranda (2007: 226) donde mencionan que existen mejores posibilidades de ocupar puestos directivos y tener crecimiento profesional dentro de la empresa. Esta situación es diferente a lo establecido en otras investigaciones como la de Bustos (2007) y Roldán et al. (2012) quienes argumentan que existen "barreras" que impiden a la mujer avanzar y ocupar cargos de mayor responsabilidad. Además, aunque las mujeres alcanzan los mismos niveles educativos que los hombres, ellas no tienen representación en los puestos de decisión dentro de la sociedad (Botello, 2014).

Con estos resultados se afirma que existe una segregación laboral (al menos en los principales puestos ocupados), la cual se fundamenta en el supuesto de que existen "trabajos de hombres" y "trabajos de mujeres" (Fernández y Martínez, 2010) en concordancia con las funciones que se desempeñan en dichas áreas. Lamas (2014), menciona que el orden simbólico de género se obtiene de estructuras sociales tales como la organización social del espacio y 
tiempo y la división sexual del trabajo. Aunado a lo anterior, la OMT (2013) y Moreno y Lunar (2006), señalan que el género determina las responsabilidades, personalidad y el sistema de valores y creencias de una persona en función del sexo, como lo establece Núñez (1999) y los niños y niñas se vinculan por separado con la madre o el padre, repercutiendo en las conceptualizaciones sobre lo que la mujer debe desempeñar laboralmente; es así que las visiones de género siguen predominando. Esto lo demuestra el hecho de que la mayoría de los varones se dediquen a actividades consideradas masculinas (mantenimiento y vigilancia) y las mujeres a las consideradas femeninas (limpieza, atención, preparación de alimentos).

Tabla 7. Distribución del personal por género de los hoteles, Toluca, México (2016)

\begin{tabular}{|c|c|c|c|c|}
\hline PUESTO & Mujeres & $\%$ & Hombres & $\%$ \\
\hline Ama de llaves & 8 & 6 & 0 & 0 \\
\hline Auxiliar de cocina & $11^{*}$ & 8 & 2 & 2 \\
\hline Auxiliar de reservas & 1 & 1 & 2 & 2 \\
\hline Barista & 1 & 1 & 0 & 0 \\
\hline Bellboy & 0 & 0 & 2 & 2 \\
\hline Camarista & $53^{*}$ & 38 & 3 & 3 \\
\hline Chef & 3 & 2 & 3 & 3 \\
\hline Chofer & 0 & 0 & 1 & 1 \\
\hline Contabilidad & 7 & 5 & 2 & 2 \\
\hline Gerente de operaciones & 0 & 0 & 1 & 1 \\
\hline Gerente general & 6 & 4 & 4 & 4 \\
\hline Jardinería & 0 & 0 & 1 & 1 \\
\hline Jefe de $A$ y $B$ & 1 & 1 & 1 & 1 \\
\hline Jefe de recepción & 2 & 1 & 3 & 3 \\
\hline Limpieza & 4 & 3 & 3 & 3 \\
\hline Mantenimiento & 1 & 1 & $23^{*}$ & 22 \\
\hline Mesero & 6 & 4 & 10 & 10 \\
\hline Otros & 9 & 7 & 9 & 9 \\
\hline Recepcionista & $18^{*}$ & 13 & $19^{*}$ & 18 \\
\hline Recursos humanos & 0 & 0 & 1 & 1 \\
\hline Steward & 3 & 2 & 0 & 0 \\
\hline Subgerente & 2 & 1 & 1 & 1 \\
\hline Ventas & 1 & 1 & 0 & 0 \\
\hline Vigilancia & 1 & 1 & $11^{*}$ & 11 \\
\hline Total & 138 & 100 & 102 & 100 \\
\hline
\end{tabular}

Fuente: Elaboración propia con base en trabajo de campo.

*Puestos con mayor número de empleados 
En relación con el ingreso mensual que perciben tanto las mujeres como hombres, $50 \%$ de los hoteles no contestaron este apartado debido a sus políticas de privacidad. No obstante, con los datos de los hoteles que sí proporcionaron esta información, se puede afirmar que las mujeres sí obtienen el mismo salario que los hombres, al menos dentro del mismo puesto. Por lo cual, en esta investigación no se cumple lo establecido por la UNWomen (2015) sobre que la mujer percibe menos dinero (entre 10 y $30 \%$ ) que los hombres, aunque desarrollen las mismas actividades; situación que es muy positiva en el caso de los hoteles de Toluca.

Del segundo cuestionario se obtuvo que $69 \%$ son madres, esto indica que más de la mitad cumple su doble papel en el país: ser madre y trabajadora (Loggia, 1998). Aunado a lo anterior, $46.6 \%$ son solteras, $37.9 \%$ casadas, $8.7 \%$ viudas y $7 \%$ vive en pareja. Con base en dichos datos, se puede establecer que el doble rol que desempeña la mujer propicia que dediquen menos tiempo a cada uno, siendo las responsabilidades familiares un factor determinante para la toma de decisiones sobre su carrera profesional (Cuadrado y Morales, 2007).

Acerca de la escolaridad, con mayores porcentajes: $18 \%$ cuenta con un nivel de estudios superior completo, $17 \%$ tiene secundaria completa, $14 \%$ preparatoria o técnica incompleta, $11 \%$ con preparatoria o técnica completa y con el mismo porcentaje, primaria completa. De acuerdo con (Hernández y García, 2015), la escolaridad es la condición previa más importante para mejorar la posición de las mujeres; en el nivel de empoderamiento se aprecia que este factor influye para que se encuentre dentro un rango mayor.

En la Tabla 8 se muestra la relación del nivel de estudios con el puesto que ocupan las mujeres, para ello se consideraron los tres puestos con mayor número de ellas, así como el de gerente general. En el caso de las camaristas, los dos porcentajes más altos (20\%) corresponden a aquellas que tienen primaria completa y secundaria completa. Por otro lado, con $40 \%$, la escolaridad de las recepcionistas fue preparatoria o técnica completa; mientras que $28.57 \%$ de las mujeres que se desempeñan como auxiliar de cocina tienen preparatoria o técnica incompleta. Finalmente, $100 \%$ de quien ocupa la gerencia general cuenta con un nivel de estudios superior completo.

De acuerdo con lo anterior, la formación superior es el último peldaño de la estructura educativa, que potencialmente ha dotado a las personas de ciertas competencias necesarias para entrar en la vida laboral. Cabe destacar que la relevancia que tiene la escolaridad en los procesos de selección y reclutamiento está llevando a que los niveles de educación mínima obligatoria sean cada vez más altos. En este contexto, se explica la relación que a mayor nivel de estudios, existen mejores posibilidades de ocupar puestos directivos o en su caso desempeñar funciones como jefe de área o departamento (Salgado y Miranda, 2007: 226). 
Tabla 8. Relación puesto - escolaridad

\begin{tabular}{|lcccc|}
\hline $\begin{array}{l}\text { Escolaridad } \\
\text { Sin estudios }\end{array}$ & Camarista & Recepcionista & Auxiliar de cocina & Gerente General \\
\hline Primaria incompleta & $2 \%$ & $0 \%$ & $14.28 \%$ & $0 \%$ \\
\hline Primaria completa & $8 \%$ & $0 \%$ & $14.28 \%$ & $0 \%$ \\
\hline Secundaria incompleta & $20 \%$ & $0 \%$ & $0 \%$ & $0 \%$ \\
\hline Secundaria completa & $18 \%$ & $10 \%$ & $14.28 \%$ & $0 \%$ \\
\hline Preparatoria o técnica incompleta & $20 \%$ & $0 \%$ & $28.57 \%$ & $0 \%$ \\
\hline Preparatoria o técnica completa & $18 \%$ & $10 \%$ & $0 \%$ & $0 \%$ \\
\hline Superior incompleta & $8 \%$ & $40 \%$ & $0 \%$ & $0 \%$ \\
\hline Superior completa & $0 \%$ & $0 \%$ & $14.28 \%$ & $0 \%$ \\
\hline Posgrado & $6 \%$ & $30 \%$ & $14.28 \%$ & $100 \%$ \\
\hline
\end{tabular}

Fuente: Elaboración propia con base en trabajo de campo.

En cuanto al salario 4 mensual, $48 \%$ percibe entre $\$ 150.00$ USD y $\$ 249.95$ USD, mientras que $35 \%$ gana menos de $\$ 149.95$ USD, $7 \%$ tiene como ingreso entre $\$ 250.00$ USD y $\$ 349.95$ USD; $4 \%$ gana más de $\$ 750$ USD; las personas que reciben entre $\$ 350.00$ USD y $\$ 499.95$ USD representan $3 \%$ al igual que aquellas que tienen un salario mayor o igual a $\$ 500.00$ USD y menor o igual a \$749.95 USD. Con base en los datos anteriores, la mayoría de las mujeres tiene ingresos mensuales bajos, recordando que las funciones que desempeñan son principalmente de camaristas, recepción y área de cocina.

En cuanto a los resultados del proceso para medir el nivel de empoderamiento de las mujeres (Tabla 5), se obtuvo con categoría "medio" los factores de igualdad, independencia, satisfacción social y temeridad. Esto significa, que son mujeres con perspectiva de equidad de género, que tienen la capacidad de tomar sus propias decisiones, sin depender de otras personas y confían en sí mismas. No obstante, pueden presentar inseguridad en sus acciones, influyendo en cómo se perciban con respecto a su entorno social; esto debido a que en un nivel medio, todavía se puede llegar a influir en ellas. En este orden de ideas es importante que sigan trabajando para tener un mayor nivel de empoderamiento.

Por el contrario, los factores con nivel "alto" fueron: influencias externas, liderazgo y seguridad; es decir, una calificación alta en estos rubros indica que el liderazgo depende en gran medida de sí mismas y no de hechos ajenos a ellas, además se consideran líderes, emprendedoras y autosuficientes. Asimismo, representan a una persona con alto grado de confianza en sí mismas y sus acciones, por lo cual buscan mejores posiciones en cualquier ámbito.

Finalmente, se sumaron las calificaciones de los factores anteriores. Retomando a Rappaport (1981 en Cano y Arroyave, 2014), existen dos niveles de empoderamiento: individual y colectivo. En este caso, se analizó un empoderamiento individual, el cual se puede ver en los siguientes resultados: el nivel de empoderamiento de las mujeres que laboran en las empresas de hospedaje en la ciudad de Toluca es "medio", lo que indica que tienen áreas de oportunidad para seguir desarrollándose y lograr un empoderamiento alto. Además, cuentan con un nivel

\footnotetext{
${ }^{4} 1$ dólar = 20 pesos mexicanos. Tipo de cambio 20/09/2016
} 
medio de fortalecimiento en su autoestima y confianza en sí mismas; es decir, tienen el poder de influir en otras personas; no obstante, todavía pueden influir en ellas.

El fortalecimiento de la identidad femenina, con el desarrollo de sus capacidades, su desenvolvimiento profesional y personal, sobre todo, auto-reconocimiento y autonomía (Aranda, 2006 en Fernández y Martínez, 2010) es de nivel medio. En este tenor, tienen la capacidad de definir sus opciones de vida y tomar sus propias decisiones (Kabeer, 1999); no obstante, la autonomía no es completa, porque de acuerdo con Pérez y Vázquez (2009), es necesario que se constituyan como individuos, separándose de su rol materno y doméstico, independientemente de la importancia de este rol en el desarrollo de la sociedad. Y por los resultados obtenidos, más del $50 \%$ de las mujeres ejerce estos dos roles en la sociedad, es así que en algunas actividades y decisiones son dependientes, reflejándose en su nivel de empoderamiento.

Acerca de su acceso para ocupar puestos de mayor poder, seis de ellas asumen el cargo de "gerente general", mientras que solo cuatro de los hombres ocupan este mismo puesto. Este hallazgo es muy interesante pues no refleja lo que sucede en otros países (Botello, 2014), en donde los puestos más importantes los obtienen varones con niveles educativos altos. En el caso de los hoteles de una estrella, se tiene registro como propietario a un hombre y en el otro hotel a una mujer. En cuanto a los demás hoteles, por el tipo de empresa (cadenas internacionales) y organigrama, solo se obtuvo información sobre el puesto de gerencia.

Tabla 9. Puesto Gerente General de los hoteles de Toluca (2016)

\begin{tabular}{c|cc|}
$\begin{array}{c}\text { CATEGORÍA } \\
\text { HOTEL }\end{array}$ & $\begin{array}{c}\text { No. de } \\
\text { mujeres }\end{array}$ & $\begin{array}{c}\text { No. de } \\
\text { hombres }\end{array}$ \\
\hline $5^{*}$ & 1 & 0 \\
$4^{*}$ & 3 & 2 \\
$3^{*}$ & 1 & 1 \\
$1^{*}$ & 1 & 1 \\
TOTAL & 6 & 4 \\
\hline
\end{tabular}

Elaboración propia.

En la Tabla 9, se observa la información sobre el número de mujeres que desempeñan el puesto de gerente, los datos se clasificaron de acuerdo con la categoría del hotel. En este sentido, el número de estrellas no es un obstáculo para que la mujer ocupe dicho cargo en la empresa. De igual forma, el nivel de estudios es un factor que favorece su participación y presencia en puestos de mayor jerarquía. No obstante, en la Figura 2, se aprecia que la mujer solo ejerce el rol de trabajadora (no de madre o esposa); esto se asocia al hecho que requiere de más tiempo y esfuerzo para llevar a cabo las actividades y responsabilidades que el puesto exige, situación que al ser también madre/esposa podría impedir su crecimiento profesional dentro de la compañía debido a los prejuicios preestablecidos cultural e históricamente, así como las barreras que la mujer se "autoimpone". 
Figura 2. Características de la mujer como gerente general en hoteles de Toluca, México 2016

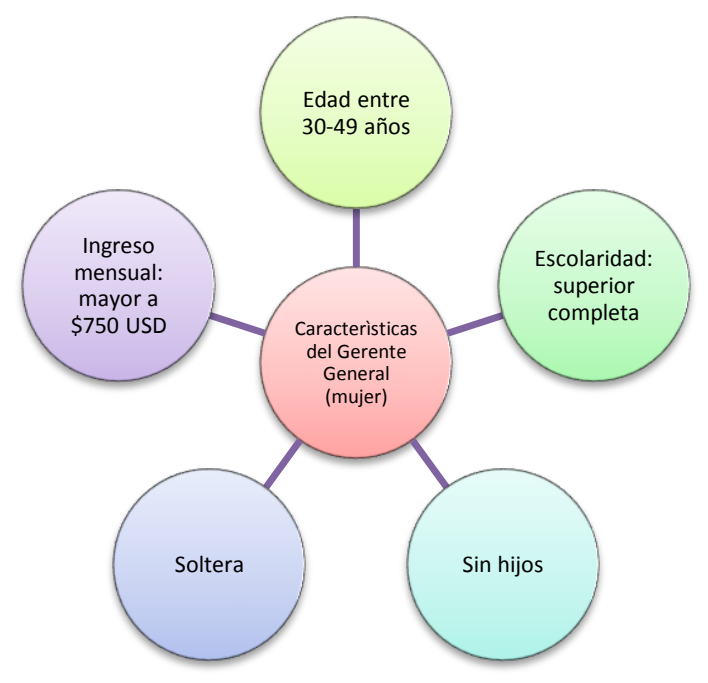

Fuente: Elaboración propia con base en trabajo de campo.

De acuerdo con los datos anteriores, es posible afirmar que las mujeres siguen incrementando su participación en puestos de esa índole. En contraposición a la existencia de "techos de cristal" o barreras "invisibles" que dificultan el acceso de las mujeres a los cargos de mayor poder, prestigio o salario, en cualquier ámbito laboral y país (Guil, 2008), en el sector hotelero de la ciudad de Toluca, no se visualiza ese tipo de barreras para acceder a puestos como la gerencia debido a que en la investigación no se trató este tema a profundidad, por lo cual los datos no son concluyentes; no obstante, se exhorta a seguir ahondado sobre el tema en futuras investigaciones.

\section{CONCLUSIONES}

Los aspectos teóricos considerados permitieron resaltar las características de esta línea de estudio poco abordada en el ámbito latinoamericano, principalmente desde un enfoque cuantitativo. En cuanto a la metodología, ésta es confiable para continuar con investigaciones relacionadas con el empoderamiento de la mujer, así como para ser utilizada en otros campos de estudio, debido a que en el análisis de confiabilidad del instrumento, éste fue positivo así como para el análisis factorial, además que esta metodología ya ha sido utilizada y supervisada en otros proyectos en México. Cabe destacar que es importante profundizar en el instrumento y realizar adaptaciones de acuerdo con el contexto a estudiar; ya que existen elementos que influyen en la obtención del nivel de empoderamiento, tal es el caso del contexto histórico y/o cultural. Por otra parte, las tres etapas de esta investigación (teórica, empírica y el contraste de resultados con los aspectos teóricos) permitieron obtener relaciones entre las variables y corroborar aspectos establecidos por los diferentes autores citados.

A partir de este trabajo se pudo constatar que se presenta una mayor participación de la mujer en el ámbito laboral, particularmente en las empresas de hospedaje de la ciudad de Toluca, Estado de México; no obstante, siguen teniendo responsabilidades relacionadas con el ámbito 
doméstico; por lo que respecta a esta perspectiva, los puestos se asignan en función del género, como camarista, limpieza y ayudante de cocina.

En contraste con lo anterior, un mayor número de ellas ocupan el puesto de gerente en los hoteles de esta investigación, en comparación a los varones; lo que se relaciona con el nivel de estudios y la preparación que tienen. En suma, aunque los procesos de empoderamiento se pueden dar a través de la educación, es importante trabajar en el desarrollo de factores como el trabajo y liderazgo, los cuales también contribuyen a dichos procesos, siendo ésta un área de oportunidad para las mujeres.

Resumiendo, la puntuación del nivel de empoderamiento se encontró muy cerca de la categoría "alto", indicando que a pesar de factores como la cultura, educación, contexto histórico, entre otros, la mujer se va fortaleciendo en su ámbito personal, teniendo más confianza en sí misma para llevar a cabo diferentes actividades en la sociedad. Igualmente, no se habla de mujeres "poderosas" por tener poder sobre sí mismas sino "empoderadas"; es decir, actúan por sí mismas, para sí mismas y demandan un trato más justo en sus lugares de trabajo, en el hogar y en el conjunto de la sociedad. Además, tienen áreas de oportunidad que pueden seguir mejorando, considerando los factores que resultaron con nivel medio: igualdad, independencia, satisfacción social y temeridad.

Lo anterior denota la importancia de dar continuidad a este tipo de análisis en investigaciones futuras relacionadas con el nivel de empoderamiento de la mujer en el sector turístico, a fin de ampliar el espectro de temas que le son inherentes a este sector cuyo abordaje había estado limitado hasta hace unas décadas a su esfera económica. Asimismo, se exhorta a realizar investigaciones desde un enfoque cualitativo para profundizar en temas relacionados con la mujer en el sector turístico. De igual forma, en la medida que la mujer tenga mayores oportunidades para participar en la sociedad, podrá repercutir e impulsar en el desarrollo no sólo a nivel familiar sino también en los diferentes ámbitos donde desempeñe algún rol.

\section{REFERENCIAS BIBLIOGRÁFICAS}

Aguilar, J.M.; Guzmán, G.; Parra, E.; Granados, A. y Rodríguez, R. (2015). Investigación sobre obstáculos para mujeres dentro del INE para acceso a cargos de dirección. Transversa Consultoría de Género y Desarrollo Social, S.C. Recuperado de: www.ine.mx/archivos3/portal/historico/.../ISU_InfRel_IGYND_Obstaculos.pdf

Araiza, A. (2004). Empoderamiento femenino: el caso de la comunidad zapatista de Roberto Barrios. Feminismo/s. 3, 135-148. Recuperado de: http://rua.ua.es/dspace/handle/10045/3238

Banco Mundial (2016). Tasa de participación en la fuerza laboral, mujeres (\% de la población femenina entre 15-64 años) (estimación modelado OIT). Recuperado de: http://datos.bancomundial.org/indicador/SL.TLF.ACTI.FE.ZS

Baum, T. (2013). International perspectives on women and work in hotels, catering and tourism. Geneve: Internationmal Labour Office. Recuperado de: http://www.ilo.org/wcmsp5/groups/public/---dgreports/--gender/documents/publication/wcms_209867.pdf 
Botello, H. (2014). Empoderamiento de la mujer latinoamericana; empleo y educación, 19602010. Tendencias \& Retos. 2, 79-99

Brunet, I. y Santamaría, C. A. (2016). La economía feminista y la división sexual del trabajo. Culturales, $\quad 4 \quad(1), \quad 61-86 . \quad$ Recuperado en: http://www.redalyc.org/articulo.oa?id=69445150003

Bucheli M. y Sanroman, G. (2005). Salarios Femeninos en el Uruguay ¿Existe un Techo de Cristal? Revista de Economía del Banco Central del Uruguay. 12 (2), 63-88. Recuperado de: https://dialnet.unirioja.es/ejemplar/241290

Buquet, A.; Cooper, J.; Mingo, A. y Moreno, H. (2013). Intrusas en la Universidad. México: Universidad Nacional Autónoma de México.

Bustos, O. (2007). Mujeres rompiendo el techo de cristal: el caso de las universidades. México: Universidad Nacional Autónoma de México. Recuperado de: http://www.posgrado.unam.mx/publicaciones/ant_omnia/41/07.pdf

Butler, J. (2007). El género en disputa: El feminismo y la subversión de la identidad. Barcelona: Paidós

Caamaño, E. (2010). Mujer y trabajo: origen y ocaso del modelo del padre proveedor y la madre cuidadora. Revista de Derecho. (34), 179-209. Recuperado de: http://www.redalyc.org/articulo.oa?id=173616611005

Cacique, I. (2008). Participación en el trabajo doméstico de hombres y mujeres en México. Papeles de población. (14), 173-200. Recuperado de: http://www.redalyc.org/articulo.oa?id=11205508

Camacho, R.; Martínez, J. y Robert, A. (2003). Mujeres en movimiento. Liderazgos Transformadores para construir buenos gobiernos en Centroamérica. Costa Rica: EUNED

Cano, T. y Arroyave, O. (2014). Procesos de empoderamiento de mujeres: subjetivación y transformaciones en las relaciones de poder. Revista Virtual Universidad Católica del Norte. 42, 94-110. Recuperado de: http://www.redalyc.org/articulo.oa?id=194230899007

Charlier, S. y Caubergs, L. (2007). El proceso de empoderamiento de las mujeres. Guía metodológica. Bélgica: Comisión de Mujeres y desarrollo. Recuperado en: http://www.dhl.hegoa.ehu.es/ficheros/0000/0251/proceso_empoderamiento_mujeres_CF D.pdf

Cobo, R. (2014). Aproximaciones a la teoría crítica feminista. Boletín del Programa de Formación. 1. Perú: Comité de América Latina y el Caribe para la defensa de los Derechos de las Mujeres (CLADEM)

Colletis, G. y Pecqueur, B. (1995). Rôle des politiques technologiques dans la creation de ressources spécifiques et d'avantages dynamiques de localisation. En A. Rallet y A. Torre (dirs.), Économie industrielle et économie spatiale. París: Economica

Connell, R. (2015), Masculinidades, UNAM - PUEG, México.

Contreras, F., Pedraza, J. E. y Mejía, X. (2012). La mujer y el liderazgo empresarial. Diversitas: Perspectivas en Psicología. 8, 183-194. Recuperado de: http://www.redalyc.org/articulo.oa?id=67923973012

Cuadrado I. y Morales. J.F. (2007). Algunas claves sobre el techo de cristal en las organizaciones. Revista de Psicología del Trabajo y de las Organizaciones. 2, 183-202. Recuperado de: http://www.redalyc.org/articulo.oa?id=231317597002 
Daros, W.R. (2014). La mujer posmoderna y el machismo. Franciscanum revista de las ciencias del espíritu. 56, 107-129. Recuperado de: http://www.redalyc.org/articulo.oa?id=343532033005

Davis, K. y Newstron J. (2004). Comportamiento humano en las organizaciones. México: McGraw Hill

Delgado, D.; Zapata, E.; Martínez, B. y Alberti, P. (2010). Identidad y empoderamiento de mujeres en un proyecto de capacitación. Ra Ximhai, Revista de Sociedad, Cultura y Desarrollo Sustentable. 3, 453-467.

Díaz, I.A., Verján, R. y Castrejón, B.E. (2014). Estereotipos de género y turismo: análisis cualitativo del "suelo pegajoso" en hoteles de 4 y 5 estrellas en Tijuana (México). TURyDes, 7 (17).

Fang, Y. (2011). Empoderamiento en los sectores comercial y de servicios. Red de Revistas Científicas de América Latina, el Caribe, España y Portugal. 9, 27-40. Recuperado de: http://www.redalyc.org/articulo.oa?id=80118612002

Ferguson, L. (2010). Turismo, igualdad de género y empoderamiento de las mujeres en Centroamérica. PAPELES de relaciones ecosociales y cambio global. 111, 123-133. Recuperado de: https://www.fuhem.es/media/cdv/file/biblioteca/PDF\%20Papeles/111/turismo_igualdad_d e_genero_y_empoderamiento_mujeres_Centroamerica_L._FERGUSON.pdf

Ferguson, L. (2011). Promoting gender equality and empowering women? Tourism and the third millennium development goal. Current Issues in Tourism, 14, 235-249. Recuperado de: http://dx.doi.org/10.1080/13683500.2011.555522

Ferguson, L. y Moreno, D. (2014). Gender and sustainable tourism: reflections on theory and practice. Journal of Sustainable Tourism. Recuperado de: http://dx.doi.org/10.1080/09669582.2014.957208

Fernández, M.; Martínez, L. (2010). Participación de las mujeres en las empresas turísticas privadas y comunitarias de bahías de Huatulco, México. ¿Hacia un cambio en el rol de género? Red de Revistas Científicas de América Latina, el Caribe, España y Portugal. 26, 129151. Recuperado de: http://www.redalyc.org/articulo.oa?id=39817020007

Fisher, L. y Espejo, J. (2011). Mercadotecnia. México: McGraw Hill

Gobierno del Estado de México (2011). Plan de Desarrollo 2011-2017. Pilar 2 Estado Progresista. México.

González, T. (2013). Según la OIT pocas mujeres ocupan cargos gerenciales en hoteles y restaurantes de Latinoamérica. Secc. Noticas de turismo y economía - indicadores turísticos. Recuperado de: https://www.hosteltur.lat/190200_pocas-mujeres-ocupan-cargosgerenciales-hoteles-restaurantes-latinoamerica.html

Guil, A. (2008). Mujeres y ciencia: techos de cristal. EccoS Revista Científica. 10, 213-232. Recuperado de: http://www.redalyc.org/articulo.oa?id=71510111

Gutiérrez, L. E. y Limas, M. (2008). Incorporación de la mujer al mercado de trabajo y desarrollo regional en Chihuahua. Estudios Fronterizos. 9, 39-70. Recuperado de: http://ww.w.redalyc.org/articulo.oa?id=53011367002

H. Ayuntamiento de Toluca (2013). Plan de Desarrollo Municipal de Toluca 2013-2015. México Hernández, J. y García, R. (2008). Instrumento para medir el empoderamiento de la mujer. México. Universidad Juárez Autónoma de Tabasco 
Hernández, J. y García, R. (2015). Empoderamiento en mujeres mexicanas: Experiencias de mujeres líderes de México. Cuestiones de Género: de igualdad y la diferencia. 10. 419-434

Hernández, M.; Cruz, G., Zizumbo, L.; Naranjo, O.; Serrano, R.; Zarza, P. (2014). Mujeres en espacios políticos. Debate en torno a la Secretaría de Turismo del Estado de México. Cultur Revista de Cultura e Turismo. 8, 114-136. Recuperado de: http://www.uesc.br/revistas/culturaeturismo/ano8-edicao3/5.pdf

INEGI (2011). Estadística de género. Proyecto Interinstitucional. XII Encuentro Internacional de Estadísticas de Género: empoderamiento, autonomía económica y políticas públicas. México: Instituto Nacional de Estadística y Geografía. Recuperado de: http://www.inegi.org.mx/eventos/2011/Encuentro_genero/doc/01-05CMEduardoSojoINEGI.pdf

INEGI (2014a). Mexicompios. Instituto Nacional de Estadística y Geografía. México. Recuperado de: http://cuentame.inegi.org.mx/mapas/pdf/entidades/div_municipal/mexicompios.pdf

INEGI (2014b). Datos censales de 2014, personal y remuneraciones por municipio del Estado de México. Instituto Nacional de Estadística y Geografía. México

INEGI (2015). Estadísticas a propósito del día mundial del turismo (27 de septiembre). Datos Nacionales. México: Instituto Nacional de Estadística y Geografía. Recuperado de: http://www.inegi.org.mx/saladeprensa/aproposito/2015/turismo0.pdf

INEGI (2016). Estadísticas a propósito del día Internacional de la mujer (8 de marzo). Datos económicos nacionales. México: Instituto Nacional de Estadística y Geografía. Recuperado de: http://www.inegi.org.mx/saladeprensa/aproposito/2016/mujer2016_0.pdf

Kabeer, N. (1999). The conditions and consequences of choice: reflections on the measurement of women's empowerment. UNRISD discussion papper no. 108. Recuperado de: http://www.unrisd.org/80256B3C005BCCF9/(httpAuxPages)/31EEF181BEC398A380256B67 005B720A/\$file/dp108.pdf

Lagarde, M. (2003). Identidad de Género y derechos humanos. La construcción de las humanas. Recuperado de: http://catedraunescodh.unam.mx/catedra/CONACYT/16_DiplomadoMujeres/lecturas/mod ulo1/2_Identidad_Genero_Lagarde.pdf

Lamas, M. (1986). La antropología feminista y la categoría "género". Nueva Antropología. 30, 173-198. México. Recuperado de: http://www.redalyc.org/pdf/159/15903009.pdf

Lamas, M. (2003). El género, la construcción cultural de la diferencia sexual. México: UNAM PUEG

Lamas, M. (2014). Cuerpo, sexo y Política, Debate. México: OCEANO

Legazpe, N. (2015). Mujer, trabajo y familia en España. En El Trimestre Económico, LXXXII (4), 873896. Recuperado de: http://www.redalyc.org/articulo.oa?id=31343926005

Loggia, S. M. (1998). Legislación laboral y participación de la mujer en el mercado de trabajo. El caso de Suecia y México: un análisis comparativo. Papeles de Población. 5, 95-125. Recuperado de: http://www.redalyc.org/articulo.oa?id=11201505

Martín-Llaguno, M. y Navarro-Beltrá, M. (2015). Vertical and Horizontal Segregation in Advertising Agencies Before and After the Law for Equality of Women and Men. Revista Española de Investigaciones Sociológicas. 150, 113-150. Recuperado de: http://dx.doi.org/10.5477/cis/reis.150.113 
Matos, M. y Paradis, C. (2013). Los feminismos latinoamericanos y su compleja relación con el Estado: debates actuales. Íconos, 45, 91-107

Mead, M. (2013). Sexo y temperamento en tres sociedades primitivas. Barcelona: Paidós, 2a․ Edición

Méndez, M. (2008). Desarrollo económico y calidad de vida en Cancún a partir del sector hotelero. Teoría y Praxis. 5, 289-304. Recuperado de: http://www.redalyc.org/articulo.oa?id=456145110021

Mendizabal, G. y López, M. (2013). Empoderamiento de las mujeres: del individual al social. Recuperado de: www.ortuella.biz/esES/servicios/igualdad/world\%20cafe\%2007052013/empoderamientodelasmujeres2013050 7.pdf

Moreno, F. y Lunar, R. (2006). Turismo Y Género: Empleo De La Mujer En La Actividad Turística De La Isla De Margarita, Estado De Nueva Esparta. PASOS Revista de Turismo y Patrimonio Cultural. 4, 373-389. Recuperado de: http://www.redalyc.org/articulo.oa?id=88140306

Murguialday, C. (2006). El largo camino hacia la equidad de género. Pueblos, (20). Recuperado de: http://www.revistapueblos.org/spip.php?article368

Núñez, G. (1999). Sexo entre varones, poder y resistencia en el campo sexual. México: El Colegio de Sonora-PUEG/UNAM

OCDE (2012). Cerrando las Brechas de Género: es Hora de Actuar México. Organización para la cooperación y el Desarrollo Económico. Recuperado en: http://www.oecd.org/gender/Closing\%20the\%20Gender\%20Gap\%20\%20Mexico\%20FINAL.pdf

Ochman, M. (2016). Políticas sociales y empoderamiento de las mujeres. Una promesa incumplida Estudios Políticos, 48, 32-51. Recuperado de: http://www.redalyc.org/articulo.oa?id=16443492003

OIT (2009). Empoderamiento de las mujeres: ¡90 años de la OIT en acción! Suiza: Oficina Internacional del Trabajo. Recuperado de: www.ilo.org/wcmsp5/groups/public/@dgreports/.../wcms_106521.pdf

OMT (2013). Informe mundial sobre las mujeres en el turismo 2010. ONU Mujeres. Recuperado de: http://www.e-unwto.org/doi/pdf/10.18111/9789284414789

ONU (2016). Progreso de las mujeres en el mundo 2015-2016. Transformar las economías para realizar los derechos. Resumen. Revista Estudios Feministas, 24 (2), 589-614. Recuperado de: http://www.redalyc.org/articulo.oa?id=38145732012

Parada, C. (2016). Empleo femenino, pobreza y desigualdad. Un análisis de microdescomposiciones, Uruguay (1991-2012). El Trimestre Económico. LXXXIII (2), 371-404. Recuperado de: http://www.redalyc.org/articulo.oa?id=31345267005

Pérez, M. y Vázquez, V. (2009). Familia y empoderamiento femenino: ingresos, trabajo doméstico y libertad de movimiento de mujeres chontales de Nacajuca, Tabasco. Convergencia. 50, 187218. Recuperado de: http://www.redalyc.org/articulo.oa?id=10511169008

Presidencia de la República (2015). Tercer Informe de Gobierno 2014-2015. Gobierno de los Estados Unidos Mexicanos: México 
Rico, M. y Gómez, J. (2009). La contribución de la mujer en la economía rural de Castilla y León. Economía Agraria y Recursos Naturales. 9, 51-77. Recuperado de: https://dialnet.unirioja.es/descarga/articulo/3115714.pdf

Roldán, E.; Leyra, B. y Contreras, L. (2012). Segregación laboral y techo de cristal en trabajo social: análisis del caso español. Portularia. 12, 43-56. Recuperado de: http://www.redalyc.org/articulo.oa?id=161024690004

Salgado, M. y Miranda, S. (2007). Mercado de trabajo y profesionistas en el Estado de México. Quivera. 9, 223-247. Recuperado de: http://www.redalyc.org/pdf/401/40190112.pdf

SECTUR (2016). Directorio de empresas de hospedaje, Toluca. México. Secretaría de Turismo Estado de México.

Sosme, M. y Casados, E. (2016). Etnia y empoderamiento: elementos para el análisis de la transformación de identidades femeninas en la Sierra de Zongolica, Veracruz. Sociológica, 31 (87), 143-173. Recuperado de: http://www.redalyc.org/articulo.oa?id=305043762005

UNESCO (1955). ¿La mujer es un ser inferior? El Correo, 11, 1-44. Recuperado de: http://unesdoc.unesco.org/images/0006/000698/069853so.pdf

UNWomen (2014). Empoderamiento político de las mujeres: marco para una acción estratégica América Latina y el Caribe (2014-2017). Entidad de las Naciones Unidas para la Igualdad de Género y el empoderamiento de las Mujeres. Recuperado de: http://www.unwomen.org//media/headquarters/attachments/sections/library/publications/2014/empoderamiento\% 20politico\%20de\%20las\%20mujeres\%20lac\%202014-17\%20unwomen.pdf?vs=1828

UNWomen (2015). Step it up for gender equality infographic. United Nations Entity for Gender Equality and the Empowerment of Women: The Beijing Platform for action turns 20. The United Nations Women. Recuperado de: http://beijing20.unwomen.org/en/infographic/beijing-at20?utm source=Say+NO+\%E2\%80\%93+UNiTE+to+End+Violence+against+Women\&utm ca mpaign=f47dbc18beMonthly+Updates+Beijing+at+20\&utm medium=email\&utm term=0 713f7be9fc-f47dbc18be-70868329\%20

Vega, P.; Santero, R.; Castro, B. y Gómez, N. (2016). Participación femenina en puestos directivos y desigualdad salarial. Un análisis en el mercado laboral español. Estudios de Economía Aplicada, 34 (1), 155-177 Asociación Internacional de Economía Aplicada. Recuperado de: http://www.redalyc.org/articulo.oa?id=30143731008

Vizcaíno, P.; Serrano-Barquín, R.; Cruz G. y Pastor M. J. (2014). Investigación turística en Latinoamérica: tendencias, aportaciones y retos. Alicante: AECIT (Asociación Española de Expertos Científicos en Turismo). Recuperado de: http://www.aecit.org/el-genero-en-lainvestigacion-y-las-politicas-turisticas-en-mexic/congress-papers/67/

Vizcaíno, P.; Serrano-Barquín, R.; Cruz G. y Pastor M. J. (2016). Teorías y métodos en la investigación sobre turismo, género y mujeres en Iberoamérica: un análisis bibliográfico. Cuadernos de Turismo. Universidad de Murcia. 38. En prensa

Wirth, L. (2001). Breaking through the glass ceiling. Women in management. International Labour Office. 